\title{
EDUCAÇÃO INTERPROFISSIONAL PARA A A INTEGRAÇÃO ENSINO- SERVIÇO-COMUNIDADE NA ATENÇÃO BÁSICA EM SAÚDE
}

\section{Angélica Lucion Farinha ${ }^{1}$; Claudia Maria Ferrony Rivas ${ }^{2}$; Naiana Oliveira dos Santos ${ }^{3}$; Juliana Silveira Colomé ${ }^{4}$}

\begin{abstract}
RESUMO
Esse trabalho tem como objetivo discutir o que a literatura existente aborda sobre o potencial da educação interprofissional para a integração ensino-serviço-comunidade na atenção básica em saúde. Trata-se de uma revisão narrativa de caráter descritivo, cuja coleta de dados foi realizada entre os meses de junho a agosto de 2021 nas bases de dados Scientific Electronic Library Online (SciELO) e Biblioteca Virtual em Saúde do Ministério da Saúde (BVS). Os artigos selecionados reportam a educação interprofissional como uma metodologia que visa a prática colaborativa entre profissionais de diferentes cursos da área da saúde. Assim, se configura como uma ferramenta eficaz para que a integração ensino-serviço-comunidade na atenção básica, a partir da inserção dos estudantes na realidade dos serviços de saúde. Considera-se que a prática interprofissional baseada na realidade das comunidades pode ser capaz de formar profissionais mais qualificados para a atenção à saúde no território das unidades básicas de saúde.
\end{abstract}

Palavras-chave: Pesquisa, Atenção à Saúde, Educação Interprofissional.

Eixo Temático: Atenção Integral e Promoção à Saúde

\section{INTRODUÇÃO}

A integração ensino-serviço-comunidade (ESC) é um dos componentes que incentiva, no Brasil, o movimento de mudanças na formação dos profissionais de

\footnotetext{
1 Acadêmica do Curso de Enfermagem - Universidade Franciscana-UFN, e-mail: angelicaluciom2011@hotmail.com

${ }^{2}$ Acadêmica do Curso de Enfermagem - Universidade Franciscana-UFN, e-mail: claudiamfrivas@gmail.com

${ }^{3}$ Professora do Curso de Enfermagem - Universidade Franciscana - UFN e-mail: naiaoliveira07@gmail.com

${ }^{4}$ Orientadora Dra. Professora do Curso de Enfermagem - Universidade Franciscana - UFN e-mail: juliana@ufn.edu.br
} 
saúde e que se constitui como uma estratégia coletiva efetiva para a qualificação do Sistema Único de Saúde,, tendo em vista que integra usuários, estudantes, professores, profissionais das equipes dos serviços de saúde e gestores. Entre os objetivos primordiais, destaca-se a qualidade da atenção prestada ao usuário, a excelência da formação profissional e o desenvolvimento dos trabalhadores dos serviços (BRITO et al., 2017).

Nos últimos tempos, a Educação Interprofissional (EIP) tem sido um dos mecanismos para efetivar a integração ESC, bem como inspirar mudanças na formação dos profissionais, tendo como objetivo principal investir na formação acadêmica para que a prática seja interprofissional. Ainda, visa a construção de ações de EIP desenvolvidas na atenção básica em saúde (ABS), favorecendo a formação de profissionais mais aptos para o trabalho colaborativo em saúde. A interprofissionalidade é definida como uma atividade que envolve dois ou mais profissionais que realizam o aprendizado de forma conjunta, de modo integrativo, com a pretensão de valorizar a qualidade da atenção prestada na saúde. Também denominada como um exemplo de formação estabelecido em ações articuladas, onde dois ou mais cursos da saúde podem contribuir para mudanças em relação a colaboração e a qualidade da atenção prestada aos usuários (BRASIL, 2018).

Neste cenário, o Programa de Educação pelo Trabalho para a Saúde (PETSaúde) Interprofissionalidade faz parte da integração de ações do plano para a implementação da Educação Interprofissional (EIP) e como potencial estratégia para contribuir com a implementação da Política Nacional de Educação Permanente, para formação e desenvolvimento de trabalhadores para o SUS. Dentre os objetivos do programa, prioriza-se a articulação entre ensino, serviço e comunidade (BRASIL, 2021).

A pesquisa tem como objetivo discutir o que a literatura existente aborda sobre o potencial da educação interprofissional para a integração ensino-serviçocomunidade na atenção básica em saúde. 


\section{METODOLOGIA}

Trata-se de uma revisão narrativa de caráter descritivo com enfoque na integração ensino-serviço-comunidade e na educação interprofissional como ferramentas para efetivação e qualificação da atenção básica em saúde. A presente revisão teve como questão pesquisa: Como a integração ensino-serviço-comunidade e a educação interprofissional podem contribuir para qualificação e efetivação da atenção básica em saúde? Como a educação interprofisisonal pode contribuir para a integração ensino-serviço-comunidade no contexto da Atenção Básica em Saúde?

A coleta de dados se realizou entre os meses de junho a agosto de 2021, e se utilizou para as pesquisas às bases de dados Scientific Electronic Library Online (SciELO), e Biblioteca Virtual em Saúde do Ministério da Saúde (BVS). Foi definido como critério de inclusão: artigos originais e escritos na língua portuguesa. Os termos utilizados para a busca dos artigos foram: Educação Interprofissional, Integração ensino-serviço-comunidade e Atenção Básica em Saúde, resultando no final um total de 17 artigos que compuseram esta revisão. Monografias, dissertações, teses e boletins informativos foram excluídos desta revisão. Após a seleção dos artigos conforme os critérios de elegibilidade previamente definidos, foram seguidos os seguintes passos: leitura exploratória, leitura seletiva e escolha do material que contemplasse os objetivos deste estudo, análise dos textos e, por último, a realização de leitura interpretativa e redação.

\section{RESULTADOS E DISCUSSÕES}

Os artigos selecionados são periódicos nacionais, no que diz respeito a caracterização dos artigos, todas as publicações foram encontradas em português, em relação ao país sede dos estudos, todos foram realizados no Brasil. Para a revisão não foi realizado restrição temporal, permitindo uma maior amplitude dos resultados para a construção do trabalho. Abaixo apresenta-se um panorama geral dos 17 estudos selecionados, destacando o que o referencial teórico existente traz sobre os temas estudados nesta revisão. 


\section{Atenção Básica em Saúde cenário para formação profissional}

A ABS é caracterizada por um conjunto de ações de saúde, no contexto individual e coletivo, que compreende ações de promoção, proteção da saúde, prevenção de agravos, diagnóstico, tratamento, reabilitação, redução de danos e a conservação da saúde que tem como objetivo desenvolver uma atenção integral que traga efeitos na situação de saúde e autonomia da população e nos determinantes e condicionantes de saúde das coletividades (BRASIL, 2017). As UBS, se constituem como um espaço efetivo para a qualificação em busca de melhor organização dos serviços e maior oferta de ações aos usuários. Para isso, é necessário profissionais com conhecimentos, habilidades e condutas específicas, capazes ao desenvolvimento de práticas de cuidado integral à saúde de forma reflexiva e construtiva, conformando equipes de atenção básica à saúde (CAVALCANTI; OLIVEIRA NETO; SOUSA, 2015).

A Política Nacional de Atenção Básica no Brasil - PNAB, estabelecida pelo Ministério da Saúde, desde 2006, é definida por um grupo de ações de saúde, no âmbito individual e coletivo, que envolve a promoção e a proteção da saúde, a prevenção de agravos, o diagnóstico, o tratamento, entre outras ações. A PNAB possui como objetivo principal desenvolver uma atenção integral que afete a situação de saúde e autonomia dos indivíduos e os determinantes e condicionantes de saúde das coletividades, por meio do exercício de práticas de cuidado e gestão participativas, de acordo com a forma de trabalho em equipe, direcionadas a populações de territórios definidos, por quais assume a responsabilidade sanitária, considerando a realidade existente no território em que vivem essas populações (BRASIL, 2017).

A ABS, é considerada um espaço privilegiado de formação para os profissionais de saúde, de acordo com os pressupostos orientadores do SUS, uma vez que se caracteriza como porta de entrada preferencial da rede de atenção à saúde, acolhendo os usuários e famílias, buscando estabelecer vínculos e responsabilização pela atenção individual e coletiva (FERTONANI et al., 2015). Para que se obtenha profissionais capacitados para atuação no SUS é necessário uma 
formação que insira os profissionais nos serviços e comunidades, diante disso a integração ensino-serviço-comunidade é uma potente ferramenta para que esse objetivo seja alcançado.

\section{Integração ensino-serviço-comunidade como ferramenta para qualificar} a formação na perspectiva interprofissional

Para o fortalecimento do SUS e da atenção básica, é preciso investir na capacitação e desenvolvimento de seus profissionais, considerados como autores das mudanças essenciais para os avanços esperados. Na criação de habilidades e atitudes, as ações educativas devem ser entendidas, para a frente do sentido modelo da aquisição de conhecimentos técnico-científicos, como um processo de formação de indivíduos críticos e reflexivos, agentes de transformação da realidade e de formação de novas formas de gestão dos processos de trabalho (BRASIL, 2017).

Diante disso, a integração ensino-serviço-comunidade, pode constituir uma oportunidade para qualificar a formação e o cuidado em saúde para o SUS, com o objetivo de alcançar a efetivação de seus princípios, como a integralidade do cuidado, e a resolutividade dos problemas e da atenção em saúde (SILVEIRA; KREMER; SILVEIRA; SCHNEIDER, 2020). Contudo a IESC trata-se de um processo complexo, tendo em vista que inclui relações individuais e coletivas, investimento e aperfeiçoamento dos profissionais e compromisso com uma estrutura acadêmica apoiada pela instituição de ensino superior com diferentes setores da comunidade de atuação teórico-prática e estágio (VENDRUSCOLO; PRADO; KLEBA, 2016). A integração ESC é um meio para que ocorra a ruptura dos modelos tradicionais de ensino, possibilitando o entendimento da importância da integralidade do cuidado. Todavia, a formação, quando associada à prática de serviço, torna-se mais significativa (CODATO et al., 2017).

Ao perceber a necessidade de contribuírem para integração entre ensino e serviços de saúde para consolidação das premissas dos SUS e para dar continuidade nas transformações da formação dos novos profissionais, os Ministérios da Saúde e da Educação têm promovido várias iniciativas para estimular a aproximação das 
Universidades aos serviços de saúde (PINTO et al., 2009). Dentre as estratégias efetivas para a integração ESC, destaca-se a criação dos programas Pró-Saúde e o PET-Saúde. Estes foram criados com vistas a introduzir a educação interprofissional nos cursos de graduação em saúde, bem como integração do ensino e serviço, de comum acordo com os preceptores e supervisores dos campos de atuação onde os estudantes estão inseridos. Tais estratégias têm o objetivo de fomentar para que a formação dos profissionais de saúde seja voltada para o cuidado integral, além de profissionais comprometidos em atender as necessidades com que poderão se deparar no serviço (CAMARA; GROSSEMAN; PINHO, 2015).

O PET-Saúde promove ações de integração ESC, com interação com a realidade, objetivando formar profissionais conscientes das necessidades que se apresentam nos serviços de saúde. Além de estimular os profissionais que já atuam nestes serviços a constantes capacitações e atualizações, reforçando a importância de serem produtores de conhecimentos e práticas, tornando-os capazes de atender às diferentes demandas da população atendida pelo SUS (BRASIL, 2012). A integração ESC, quando adequadamente planejada, promove ganhos a todos os sujeitos envolvidos, sejam universidades, professores, estudantes, profissionais e usuários. Por isso, de maneira coletiva, todos devem realizar esforços para que ela, com toda a sua potencialidade, se efetue (FRANCOS, et al., 2020).

A integração ESC é também considerada uma ferramenta para efetivação da educação interprofissional, tendo em vista que os estudantes ao adentrarem em serviços de saúde por meio da integração ESC tem a oportunidade de atuar em equipes de saúde e por meio disso criar estratégias ou incentivar as já existentes, que tem como objetivo a implementação da educação interprofissional. As estratégias de educação interprofissional no panorama da integração ESC são ferramentas para que o cuidado à população seja realizado de forma resolutiva e integral, tendo em vista a prática colaborativa, onde dois ou mais profissionais de cursos diferentes atuam de forma conjunta em busca do melhor para o usuário.

Educação Interprofissional em Saúde para a formação do trabalho em equipe 
$\mathrm{Na}$ perspectiva interprofissional, o tema da educação em saúde tem sido acompanhado por debates sobre as mudanças no ensino superior há alguns anos. No que diz respeito ao Brasil, com o debate sobre a integralidade da assistência, especialmente no berço da Reforma Sanitária, que mobilizou fortemente a formação e o trabalho em saúde, fez com que se tornasse mais evidente o esgotamento da perspectiva da uniprofissionalidade. O processo de busca por um sistema de saúde que atenda às diferentes necessidades de saúde de forma integral e universal, solicita mudanças importantes de discussão do modelo de atenção e formação em saúde. Para pensar em um novo modelo de atenção à saúde como exercício da cidadania, com respaldo do conceito ampliado em saúde, requer pensar o trabalho em saúde, que deve ter como foco a lógica do trabalho em equipe (TOASSI, 2017).

A educação e a prática interprofissional têm uma importância única no contexto Política Nacional de Saúde, com base no reconhecimento da premissa de que o Sistema Único de Saúde (SUS) é interprofissional, confirmando que seus princípios possuem ligação com a educação interprofissional (EIP), principalmente no que tange à atenção básica à saúde, que através da Estratégia Saúde da Família (ESF), que traz a reorientação e que envolve diferentes profissões em equipe para a atuação integrada (PEDUZZI, 2016).

A interprofissionalidade está sendo cada vez mais discutida junto às políticas de saúde, no que concerne ao setor público e privado, no país e no mundo, tendo como referência a educação interprofissional. Esta importância é resultado da relevância de inserir na gestão do processo de trabalho e da educação na saúde métodos e processos de sistematização da prática profissional em equipe e a formação profissional para o trabalho em equipe. A discussão necessária, portanto, não está apenas atrelada aos métodos e processos, o conceito e sua trajetória ainda são pouco discutidos e usá-lo, na prática do trabalho ou da formação, ainda vem permeado pelo desconhecimento, quando não tratados apenas como simples sinônimo das palavras "multiprofissionalidade", "multidisciplinaridade" e "interdisciplinaridade" (CECCIM, 2018).

Para compreender a interprofissionalidade é necessário estar inserido e observar o trabalho em equipe de saúde, a realidade das práticas, assim é possível 
que os acadêmicos e profissionais entendam a sua complexidade e transcendam a concepção minimalista de que, para realizar o trabalho interprofissional, 'basta existir vários profissionais agrupados em uma mesma unidade de saúde. A integralidade do cuidado é alcançada através do diálogo e colaboração entre os profissionais, que só se realiza quando há interação, confiança, determinação de papéis e uma liderança colaborativa e participativa (SALOMÃO, et al., 2018).

No que tange a Educação Interprofissional essa se difere da educação profissional tradicional, pois a geração de conhecimento se dá a partir da integração com os demais profissionais e envolve atitudes e práticas colaborativas singulares, e, por isso, ela necessita de um novo jeito de pensar o processo de ensinoaprendizagem. No Brasil, entre os dispositivos para incorporar a Educação Interprofissional na formação na área da saúde, salienta-se o Programa de Educação pelo Trabalho para a Saúde do Ministério da Saúde (PET-Saúde), (BRASIL, 2008), com intuito de incentivar grupos interprofissionais de aprendizagem para o trabalho, tendo como propósito à formação dos profissionais da saúde para um aprendizado integrativo, primordial para a integralidade do cuidado, um dos princípios doutrinários do Sistema Único de Saúde (SUS). O objetivo é aproximar o acadêmico dos processos de trabalho comuns a todos os profissionais de saúde, não só aqueles específicos de sua área de formação, no período que vai de um a dois anos. Essa proximidade ocorre em grupos de acadêmicos de diferentes cursos e proporciona vivências interprofissionais (CAMARA; GROSSEMAN; PINHO, 2015).

A prática colaborativa ocorre quando diferentes profissionais de saúde, com distintas experiências profissionais, realizam o trabalho em saúde envolvendo paciente, família, cuidador, e comunidade em busca do cuidado integral, os profissionais da saúde integram os usuários, onde as suas habilidades podem ajudar na obtenção dos objetivos de saúde local (OMS, 2010). Desta forma torna-se evidente que as ações e programas de extensão que são fundamentados na interdisciplinaridade e na interprofissionalidade propiciando trocas de vivências entre os estudantes e as comunidades, construindo relações horizontais nos encontros entre os conhecimentos populares e científicos e o empoderamento dos indivíduos, a fim de superar as desigualdades sociais (RIOS; SOUSA; CAPUTO, 2019). 
Diante disso a atenção básica se configura como um campo relativamente estratégico e com potencial acentuado para a integração ESC bem como a educação interprofissional, tendo em vista como citado anteriormente no estudo que as Unidades Básicas de Saúde e as Estratégias de Saúde da Família são potenciais áreas de ensino aprendizagem que tem como objetivo fortalecer estes aspectos visando a formação de profissionais capacitados para atuar de modo colaborativo, envolvendo o ensino, o serviço e a comunidade em prol da assistência de qualidade, integral e resolutiva de acordo com os princípios doutrinários do SUS.

A educação interprofissional é uma metodologia que tem como objetivo a prática colaborativa entre profissionais de diferentes cursos da área da saúde, se configura desta forma como uma ferramenta eficaz para que a integração ensinoserviço-comunidade seja realizada de forma que potencialize a formação baseada na realidade dos serviços de saúde, favorecendo aos estudantes a prática baseada na realidade das comunidades, o que reproduz profissionais mais capacitados no atendimento a população adiscrita nas unidades básicas de saúde.

\section{CONCLUSÃO}

Este estudo permitiu compreender aspectos relacionados à integração ensinoserviço-comunidade na atenção básica e a importância da educação interprofissional para qualidade da assistência prestada. Esta revisão aponta que a integração ensinoserviço-comunidade é uma importante ferramenta para a qualificação profissional e para a resolutividade dos problemas da população, no entanto a educação interprofissional ainda se configura como um desafio a ser implementado seja pela resistência dos profissionais ou muitas vezes pelo desconhecimento dos mesmos sobre a educação interprofissional, muitos avanços já foram alcançados mas ainda é necessário que a educação interprofissional seja pauta nas metodologias de ensino das graduações em saúde bem como residências, mestrados entre outras especialidades, para que assim se obtenha o cuidado pautado na perspectiva interprofissional, de forma singular e integral. 


\section{AGRADECIMENTOS}

A Universidade Franciscana-UFN e a professora orientadora.

\section{REFERÊNCIAS}

BRASIL. Ministério da Saúde. PET-Saúde Interprofissionalidade. As Contribuições do PET-Saúde Interprofissionalidade para a reorientação da formação e do trabalho em saúde no Brasil. Brasília-DF, 2021.

BRASIL. Ministério da Saúde. Secretaria de Atenção à Saúde. Departamento de Atenção Básica. Política Nacional de Atenção Básica. Brasília, DF: Ministério da Saúde, 2012.

BRASIL. Ministério da Saúde. Portaria Interministerial no 1.802, de 26 de agosto de 2008. Institui o Programa de Educação pelo Trabalho para a Saúde - PET-Saúde. Brasília-DF, 2008.

BRASIL. Ministério da Saúde. Portaria no 2.436, de 21 de setembro de 2017. Aprova a Política Nacional de Atenção Básica, estabelecendo a revisão de diretrizes para a organização da Atenção Básica, no âmbito do Sistema Único de Saúde (SUS). Brasília, DF, 2017.

BRASIL. Ministério da Saúde. Secretaria de Gestão do Trabalho e da Educação na Saúde. Departamento de Gestão da Educação na Saúde. Política Nacional de Educação Permanente em Saúde: o que se tem produzido para o seu fortalecimento? Brasília-DF, 2018.

BRITO, M.C.C. et al. Formação do enfermeiro para a atenção básica: um olhar sobre o conhecimento produzido. SANARE, Sobral, v. 16, n. 2, p. 93-102, jul./dez. 2017. 
CAMARA, A.M.C.S.; GROSSEMAN, S.; PINHO, D.L. Educação interprofissional no programa Pet-Saúde: a percepção de tutores. Interface (Botucatu), v. 19, supl. 1, p. 817-829, 2015.

CAVALCANTI, P. C. S.; OLIVEIRA NETO, A. V.; SOUSA, M. F. Quais são os desafios para a qualificação da Atenção Básica na visão dos gestores municipais? Saúde em Debate, v. 39, n. 105, p.323-336, abr./jun. 2015.

CECCIM, R.B. Conexões e fronteiras da interprofissionalidade: forma e formação. Interface v. 22, Supl. 2, p. 1739-1749, 2018.

CODATO, L. A. B. et al. Estudantes, docentes e profissionais na atenção básica: coexistência segundo a fenomenologia heideggeriana. Trabalho, Educação e Saúde, v. 15 n. 2, p. 519-536, maio/ago. 2017.

FERTONANI, H. P. et al. Modelo assistencial em saúde: conceitos e desafios para a atenção básica brasileira. Revista Ciência \& Saúde Coletiva, v. 20, n. 6, p. 18691878, 2015.

FRANCO, E.C.D. et al. A Integração Ensino Serviço Comunidade no curso de Enfermagem: o que dizem os enfermeiros preceptores. Enferm. Foco, v. 11, n. 3, p. 35-38, 2020.

Organização Mundial da Saúde. OMS. Marco para a ação em educação interprofissional e prática colaborativa. Brasília-DF, 2010.

PEDUZZI, M. O SUS é interprofissional. Interface comum. Saúde educ., v. 20, n. 56, p. 199-201, 2016.

PINTO, M. E. B et al. Experiência interdisciplinar em equipe multiprofissional na graduação na atenção primária a saúde, PET-saúde UFCSPA. In.: Congresso Multidisciplinar de Saúde Comunitária do MERCOSUL, 4. Anais. Gramado: Universidade Federal de Ciências da Saúde de Porto Alegre, 2009. 


\section{QuFN}

RIOS, D.R.S.; SOUSA, D.A.B.; CAPUTO, M.C. Diálogos interprofissionais e interdisciplinares na prática extensionista: o caminho para a inserção do conceito ampliado de saúde na formação acadêmica. Interface, v. 23, e180080, 2019.

SALOMÃO, A.F.S. et al. Educação interprofissional no contexto da atenção primária à saúde: relato de experiência. Rev. APS., 21, n. 4, p. 747 - 756, 2018.

SILVEIRA, J.L.G.G.; KREMER, M.M.; SILVEIRA, M.E.U.C.; SCHNEIDER, A.C.T.C. Percepções da integração ensino-serviço-comunidade: contribuições para a formação e o cuidado integral em saúde. Interface, v. 24: e190499, 2020.

TOASSI, R.F.C. Interprofissionalidade e formação na saúde: onde estamos? Série vivência em educação na saúde. 1 ed. Editora Rede Unida, 2017.

VENDRUSCOLO, C.; PRADO, M.L.; KLEBA, M.E. Integração ensino-serviço no âmbito do Programa Nacional de Reorientação da Formação Profissional em Saúde. Revista Ciência \& Saúde Coletiva, Rio de Janeiro, v. 21, n. 9, set. 2016. 